\title{
Adaptation to simulator sickness in older drivers following multiple sessions in a driving simulator
}

\author{
Isabelle Mackrous $\mathrm{PhD}^{\mathrm{a}}$ \\ Martin Lavallière $\mathrm{PhD}^{\mathrm{a}}$ \\ Normand Teasdale PhDa
}

\begin{abstract}
a Département de kinésiologie, Faculté de médecine, Université Laval, Québec, Canada; E: isabelle.mackrous@kin.ulaval.ca
\end{abstract}

\begin{abstract}
I. Mackrous, M. Lavallière, N. Teasdale, Adaptation to simulator sickness in older drivers following multiple sessions in a driving simulator. Gerontechnology 2014;12(2):101-111; doi:10.4017/gt.2013.12.2.004.00 There is a large proportion of individuals experiencing simulator sickness symptoms (headaches, nausea, paleness, etc.). Previous studies have reported that driving in a simulator, compared to on-road driving, can alter braking responses while stopping at intersections. Here we evaluated whether altered braking responses observed in fixed-base simulators could be linked to the presence of sickness symptoms in older drivers. Older individuals participated in a 5-session training study that included a pretest, three training sessions and a post-test. We evaluated adaptation to simulator sickness through repeated exposure (5 simulator sessions). Furthermore, we assessed whether head postural instability accounted for the presence of sickness symptoms. Sickness symptoms were measured with the Simulator Sickness Questionnaire (SSQ) and a Visual Analog Scale (VAS). After the first session, half of the participants reported mild symptoms (MS, VAS score $>5$ ), and another half reported light symptoms (LS, VAS score $<5$ ). We compared sickness symptoms, braking responses and postural stability between the two groups for the first (pre-test) and last (post-test) driving sessions. During the pre-test, participants of the MS group braked earlier, showed longer time of deceleration, a greater number of pedal activations and showed increased irregularities in their deceleration profile. Participants from the MS group adapted in that, sickness symptoms reduced through exposure. During the post-test, we observed a larger decrease in variability for the MS than the LS group, but we did not find any difference regarding the braking responses per se or the head postural stability. We interpreted these results as evidence that adaptation is accompanied with a reduction of variability. Finally, we propose that a more gradual exposure to simulated environment might be required before evaluating the driving performance of individuals prone to simulator sickness.
\end{abstract}

Keywords: driving simulator, simulator sickness, adaptation, braking responses

Mid- and low-range driving simulators raise growing interest among safety agencies worldwide. They are well suited for large-scale deployment and allow presentation of a wide range of driving contexts in a safe environment ${ }^{1}$. In several countries, general driving training and assessment protocols already integrate the simulator environment in their method ${ }^{2,3}$ and it has been argued that in certain circumstances, they provide more sensitive measures of driving efficiency than road tests $^{4-8}$. Because training and retraining of older drivers is a topic of growing interest, it appears important to document more effectively how older drivers respond to simulators before simulator solutions can be implemented at a greater scale.

Currently, there are suggestions that, when exposed to a simulator environment, there is a large proportion of individuals experiencing a set of undesirable physiological reactions (headaches, nausea, paleness, etc.) known as simulator sickness ${ }^{9}$. There are suggestions that older drivers are more sensitive than younger individuals leading to higher attrition rate in older driv$\mathrm{ers}^{10,11}$. Furthermore, simulator sickness symptoms could have adverse consequences on training as it could alter the training by distracting the learner from the primary task or even encourage the learner to adopt/learn incorrect behaviors to avoid symptoms ${ }^{12,13}$. For instance, a participant may choose to reduce normal head movements when driving in a simulator because head movements accentuate sickness symptoms ${ }^{14}$.

One hypothesis to explain motion and simulator sickness is that it arises from a sensory conflict ${ }^{15}$. The absence of expected sensory signals or the presence of contradictory sensory signals originating from different modalities would lead to sickness symptoms. For instance, while decelerating a vehicle, visual, vestibular and kinesthetic information specific to a deceleration are expected. In the particular case of braking into a fixed-based simulator, visual information informs 


\section{Simulator sickness in older drivers}

the driver that he is decelerating while the vestibular system does not perceive any deceleration or motion. This discrepancy between visual and vestibular signals (and certainly kinesthetic signals as well) could contribute to create the type of conflict underlying simulator sickness. The role of visuo-vestibular conflict in provoking simulator sickness was also supported by studies demonstrating that patients with bilateral labyrinthine defects resist to simulator sickness ${ }^{15-17}$. According to this theory, symptoms may persist until a new coupling between actions and the expected sensory consequences is established. For instance, Reason and Brand ${ }^{15}$ suggested this could explain why navy sailors adapted to a destroyer get seasick again, and must readapt again, to the different motions of an aircraft carrier or ocean liner.

In another theory proposed by Riccio and Stoffregen ${ }^{18}$, the postural instability theory of motion sickness, suggests that nausea-inducing situations are characterized by novel demands on the postural control for which the participant does not possess effective strategies, which leads to instability. For example, individuals might get seasick because maintaining upright stance when standing on a wobbly ship necessitates different control strategies when compare to standing on stationary surfaces. Stoffregen et al. ${ }^{19,20}$ noted that postural instability can also predict visually induced motion sickness. When exposed to a visual stimulus (e.g., a small sinusoidal motion of walls in a room), participants that expressed motion sickness exhibited an increased postural motion prior the onset of sickness symptoms. Postural instability may serve as reliable predictors of motion and simulator sickness and suggests that the symptoms may persist until new patterns of motor actions are established.

Compared to on-road driving, driving in a fixedbase simulator alters some driving behaviors ${ }^{21-23}$. Of particular interest to the present paper, there are studies reporting difficulties in controlling the deceleration of the vehicle in a fixed-base simulator $^{22,24-28}$. This was evidenced by the observation that drivers generally start braking earlier in a fixed-based simulator than in real-world driving. Moreover, the speed-time profiles in the simulator were characterized by clear corrections in the slope of the speed curves which resulted from participants pressing the brake, releasing the brake and letting the 'car' going at constant speed and pressing the brake again before coming to a full stop. For instance, Boer et al. ${ }^{25}$ showed that participants exhibited constant deceleration rates when making braking-tostop maneuvers in a real vehicle whereas they showed multi modal braking profiles in a driving simulator. This also was exemplified in Siegler et al. ${ }^{28}$, who showed that drivers exhibited higher jerk (second derivative of the deceleration profile representing changes in deceleration) at the onset of the deceleration and higher maximal deceleration rate when driving in a fixed-based rather than in a motion-based simulator. Furthermore, at the end of the deceleration, drivers ended farther from the target when driving in the fixed-based simulator. Although these effects apparently were observed regardless of simulator sickness symptoms, it remains to be determined whether altered driving behavior observed in fixed-base simulators could be linked to the presence of sickness symptoms in older drivers.

Recently, we had older drivers who participated in a 5-session learning study in a fixed base simulator $^{29}$. After every session, participants completed the Simulator Sickness Questionnaire (SSQ) ${ }^{30}$ and a Visual Analog Scale (VAS) to evaluate if they felt any simulator sickness symptom. Overall, 16 participants completed the entire protocol (5 simulator sessions over a 2-week period). For these participants, we noted a dichotomy in the severity of the symptoms after the first simulation session with half of the participants reporting hardly any symptoms and the other half reporting mild symptoms.

The first goal of the present paper was to conduct a complementary analysis to determine if participants who were more sensitive to the simulator sickness exhibited different postural (documented from head movements) and braking responses than those showing less sensitivity. Second, we wanted to determine whether adaptation (reduced symptoms and action responses changes) would take place for the participants who reported more pronounced simulator sickness symptoms. To this end, participants were assigned to one of two groups according to the severity of their symptoms after the first simulator session. The present study examined if the magnitude of postural (head movements) and the irregularities of braking responses could be linked with the presence of more severe symptoms observed for the first session and if this was the case to determine the effect of repeated exposure on the sickness symptoms, the head postural responses and the braking responses.

\section{Method \\ Participants}

Twenty two participants (age range 65-84 years old) participated on a voluntary basis in a learning study. All participants were active and healthy drivers. Upon their arrival in the laboratory, each participant was briefed on the requirements of the experiment and all read and signed an informed consent declaration conformed to the In- 


\section{Simulator sickness in older drivers}

stitutional Review Board. Clinical tests (MMSE) ${ }^{31}$, Snellen visual acuity, Melbourne Edge test, ankle proprioception acuity ${ }^{32}$, lower limb touch thresholds measured with a Semmes-Weinstein pressure aesthesiomether ${ }^{33}$ served to screen for impairments that might affect driving and cognition. All drivers scored 27 or higher on the MMSE and had normal or corrected-to-normal vision. They also completed the Motion History Questionnaire $(\mathrm{MHQ})^{34}$ and none reported previous history of motion sickness. Answers to the MHQ before the first session revealed that only one participant reported previous exposure to a driving simulator (one session, two years previous to the actual study). Before and after each experimental run, subjects completed a $10 \mathrm{~cm}$ visual analog scale (VAS) about their nausea symptoms $(0=$ no symptom, $10=$ mild nausea $)$ and the simulator sickness questionnaire (SSQ: 16 symptoms rated on a 4-point scale: $0=$ absent, $1=$ slight, $2=$ moderate, $3=$ severe). Participants who, after the first experimental run, felt no or light symptoms of simulator sickness (VAS score $<5$ ) were included in a light symptoms group (LS) and participants who felt more pronounced symptoms of simulator sickness (VAS score $>5$ ) were included in a mild symptoms group (MS). No medication to prevent motion sickness was taken by participants prior to the simulator sessions, and none of the participants reported long lasting simulator sickness symptoms after the initial session. Out of those 22, two participants elected to stop their participation after the first practice period with the simulator. One participant completed the first session only and data for three others participants were not complete because of overheating problems in the laboratory. Data for the remaining sixteen individuals are reported herein.

\section{Simulator}

The simulator consisted of a fixed-based opencab powered by STISIM Drive 2.0 (System Technology Inc., Hawthorne, California, USA). Imag- es were projected on a flat wall (1.45 m highx2.0 $\mathrm{m}$ wide) located $2.2 \mathrm{~m}$ from the steering wheel using a liquid crystal display projector (Hitachi CP-X275). The projector displayed a $40^{\circ}$ horizontal by $30^{\circ}$ vertical field-of-view. The center of the screen was located at the eye-level through the midline of the subject. Refresh rate varied between $25-30 \mathrm{~Hz}$ depending upon the density of the visual information displayed. During testing, ventilation was provided by a ceiling vent positioned just above the driver and, for the current study the average temperature within the room was $19.5^{\circ} \mathrm{C}(\mathrm{SD}=0.6)$.

Head movements (medio-lateral and antero-posterior displacements) were recorded using a magnetic tracker [Flock of Birds, Ascension Technology Corporation, Burlington, Vermont, USA] fixed on a head band at a frequency of $60 \mathrm{~Hz}$.

\section{Procedures}

Participants were exposed to five simulator sessions presented on separate days within a 14-day period (a pre-test, a post-test and three training sessions). On average, the time interval between each simulator session was 2.4 days $(\mathrm{SD}=1.7)$. Before the first session, participants were made aware the simulator could make them feel uncomfortable (nausea, dizziness, general discomfort, and headache). They were instructed specifically to inform the experimenter if this happened and were told to stop the simulation session before they felt discomfort or illness that could lead to emetic responses. As mentioned above, two participants elected to withdraw from the study. Before each session, participants were first asked if they were in their usual state of fitness (that is, not suffering from a cold or flu, hangover, etc.). Then, they completed the SSQ and the $10 \mathrm{~cm}$ VAS about their nausea symptoms. Then, they were exposed to a $6 \mathrm{~km}$ practice run (with less graphical information than the experimental scenario) serving the purpose of familiarizing subjects with the simulator. They filled a VAS score immediately after this practice before a 5 min rest was provided. The experimental run followed. For the first (pre-test) and last sessions (post-test), a continuous $26 \mathrm{~km}$ scenario of urban and rural roads with minor grade changes was presented. To comply 


\section{Simulator sickness in older drivers}

with the $40^{\circ}$ field of view limitation of our simulator, there was no right or left-turn maneuver at intersections. Moderate curves only were presented (smallest radius of $120 \mathrm{~m}$ ). The scenario included a normal driving environment (two-way and four-way roads, intersections with stop sign or crossing light) and specific recorded instructions to overtake slower moving vehicles. Lane width and markings were according to governmental rules and speed limits and advisory signs appeared throughout the scenario. Subjects were asked to comply with local traffic laws throughout the course of the experiment. The scenario did not require any emergency braking response unless a driving error was made by the driver. Five intersections, for which drivers had to immobilize the vehicle, were included within the scenario (intersection 1 to 5). There was no car preceding the participant's vehicle at these intersections. The breaking responses at these intersections were used to test our hypotheses. Session 2 to 4 were training sessions with specific feedback on the driving performance. These training sessions varied in length from subject to subject but were shorter than the pretest and the post-test sessions (on average, $16 \mathrm{~km}$ vs. $26 \mathrm{~km}$, respectively). After each experimental run, subjects completed again the VAS and the SSQ. Hence, VAS scores were taken before the practice run, after the practice run and after the experimental run; VAS scores taken after the experimental run served to evaluate simulator sickness symptoms. SSQ scores were taken before the practice run and after the experimental run.

\section{Data analysis: Postural and braking responses}

Head postural responses and braking responses for five intersections that required participants to bring the vehicle to a complete stop were analyzed. The head postural stability was calculated during a period of 40 seconds preceding each intersection. The range of motion was calculated as the area comprise within an ellipse that regrouped $95 \%$ of the head planar displacement (medio-lateral and antero-posterior head displacement; $x$ and $y$, respectively). Displacement variability was calculated and was defined as the standard deviation of the resultant head planar displacement $\left(R_{H D}\right)$.

$$
R_{H D}=\sqrt{ }\left(x^{2}+y^{2}\right)
$$

To document the braking responses, four variables were measured. When approaching an intersection, participants have to estimate the time they will arrive at the stop line in order to decide when to apply the brake. The time to reach the intersection evaluates this behavior, and it is calculated as the time between the moment participants first touched the brake pedal and the arriv- al at the stop line if participants had maintained a constant speed. Time of deceleration is the time from the first contact with the brake pedal to immobilization of the vehicle. Smoothness of the deceleration can be characterized by the number of changes in the jerk-time profiles (second derivative of the velocity) and by the number of gas and brake pedals activation, with a smaller number of pedals activation generally indicating a smoother deceleration profile. Specifically, for each intersection, we computed the number of peaks with values exceeding $3 \mathrm{~km} / \mathrm{h}^{3}$ in the jerk time-profile and the number of time that the gas and brake pedals were activated when decelerating. Finally, the absolute difference between the position of the vehicle (when the velocity first reached $0 \mathrm{~km} / \mathrm{h}$ ) and the position of the stop line was calculated (absolute spatial error). Within-participant variability (SD) also was computed on those variables.

\section{Statistical analyses}

For each group, VAS and SSQ scores were initially submitted to separate statistical analyses because participants were assigned to the LS or MS group based on their VAS score at the end of the first session (LS group, VAS $<5$; MS group, VAS $>5$ ). For each group, SSQ scores were submitted to a Session(five sessions)xPeriod (baseline, end of the session) analysis of variance (ANOVA) with repeated measures on both factors. Similarly, data for the VAS were submitted to a Session(five sessions)xPeriod (baseline, post-familiarization, end of the session) ANOVA with repeated measures on both factors. Because session 2 to 4 were training sessions, driving data for session 1 (pre-test) and 5 (post-test) only were analyzed. This was made necessary because driving was regularly interrupted to provide feedback about the driving performance during the training sessions. Postural variables (head movements) were submitted to ANOVAs contrasting Group(LS and MS)xSession (pre-test and post-test)xIntersection (intersection 1 to 5) with repeated measures on the last factor. For the braking responses, when data were normally distributed, they were submitted to ANOVAs contrasting Group (LS and MS)xSession (pre-test and post-test) with repeated measures on the last factor. Alternatively, when the data did not show normal distribution (e.g., number of time the gas and the brake pedals were activated), non-parametric statistic were performed (i.e., Mann-Withney $U$ test and Wilcoxon Match-Pairs Signed rank, W test). SPSS version 13.0 was used to run statistical analyses.

\section{RESULTS \\ Adaptation to simulator sickness}

Overall, it shows that scores for the SSQ and the VAS were higher for the MS than for the 


\section{Simulator sickness in older drivers}

LS group (Figure 1). First, for the LS group, the ANOVA for the SSQ score showed main effects of Session: $(F(4,24)=9.70, \quad p<0.001)$, Period: $(\mathrm{F}(1,6)=6.91, \mathrm{p}<0.05)$ and a significant interaction: $(F(4,24)=4.89, p<0.01)$. For the VAS, none of the effects were significant $(p>0.05)$. Overall, this confirms that the LS group experienced light symptoms across all sessions. Nevertheless, for the first two sessions, the SSQ scores were higher at the end of the sessions than at the beginning. Also, the score at the end of the session decreased gradually from pre-test to post-test (on average, 40.5, 18.2, 13.5, 8.4, and 9.3 for the pre-test, Training session 2 to 4 and post-test, respectively). For the MS group, the ANOVA for the SSQ scores showed significant main effects of Session: $(F(4,24)=7.88, p<0.001)$, Period: $(F(1,6)=16.44, p<0.01)$ and a significant interaction of Session by Period: $(F(4,24)=9.63$, $\mathrm{p}<0.001)$. For the VAS, a main effect of Session: $(F(4,8)=6.31, p<0.05)$ and a significant interaction of Session by Period: $(F(8,16)=4.89$, $\mathrm{p}<0.01)$ also were observed. Scores at baseline were not different across all sessions $(p>0.05)$. Through repeated exposure to the simulator, the MS group showed a gradual decrease of their symptoms at the end of the session, (on average for the SSQ score, 100.7, 48.1, 31.5, 16.0, and 37.4 for the pre-test, Training session 2 to 4 and

Group LS (Light Symptoms)
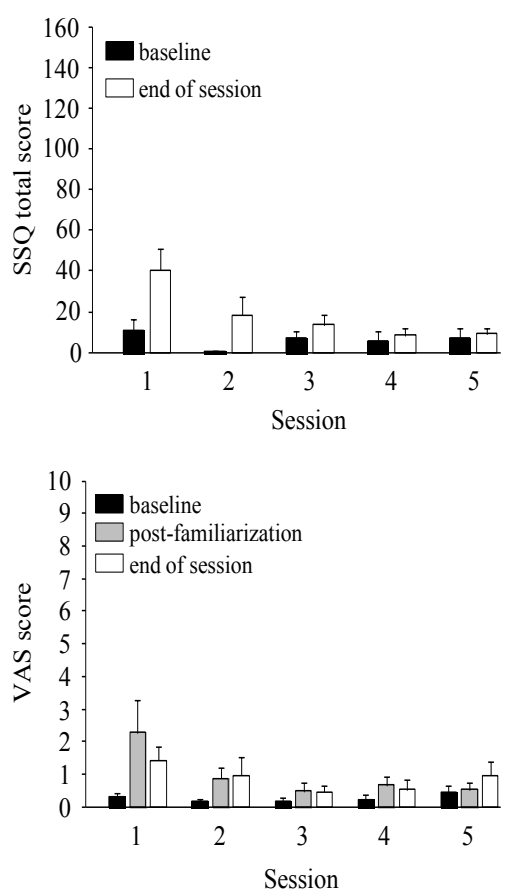

post-test, respectively). The significant increase of the SSQ score between Session 4 and posttest $(p<0.05)$ is likely associated to the longer duration of the simulator exposure for the post-test (on average, participants drove a little more than $26 \mathrm{~km}$ for session $5 \mathrm{vs}$. $16 \mathrm{~km}$ only for session 4 ). A Group by Period comparison of the SSQ score for the post-test only showed the scores before exposure were not different between groups (on average, 7.5 and 9.3 for the LS and MS groups, respectively; $p>0.05)$. On the other hand, at the end of the session, the MS group showed a higher SSQ score than the LS group (38.3 vs. 11.7, respectively; $p<0.01$ ). A similar analysis for the VAS showed that the scores were higher for the MS than the LS group for all three measurements (baseline, after familiarization, and end of session) and that both groups showed a small increase between the baseline and the end of the session scores (for both groups, an increase of 1.1 in the VAS from the baseline to the end of the session; $\mathrm{p}<0.05)$.

\section{Head postural stability}

During the pre-test, the mean area comprise within an ellipse regrouping $95 \%$ of the head displacements was of $6.8 \pm 1.6 \mathrm{~cm}^{2}$ and $12.7 \pm 4.9 \mathrm{~cm}^{2}$ for the LS and the MS groups, respectively. During the post-test, mean area was of $12.5 \pm 4.9$ and $12.3 \pm 3.0$ for the LS
Group MS (Mild Symptoms)
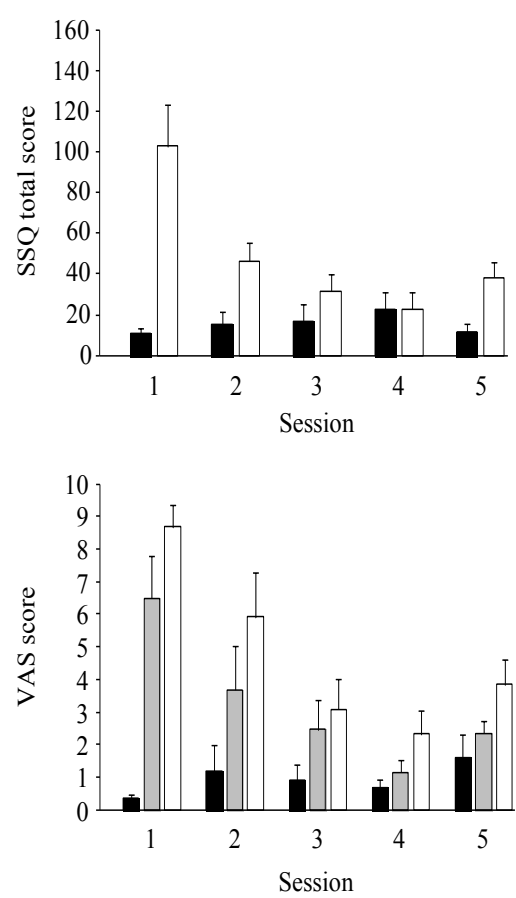

and the MS groups, respectively (not illustrated). Variability of head displacements was of $0.7 \pm 0.11 \mathrm{~cm}^{2}$ for the LS group and $0.8 \pm 0.15 \mathrm{~cm}^{2}$ for the MS groups during the pre-test (not illustrated). During the post-test, similar head displacement variability was observed for both groups [LS group: $0.8 \pm 0.2$ and MS group: 0.9 \pm 0.2 ]. The ANOVA for both the area and the variability did not reveal any significant main effect of Group, Session, Intersection or any interaction between factors $(p>0.05)$.

Figure 1. SSQ (Simulator Sickness Questionnaire) and VAS (Visual Analog Scale) scores for both groups, LS (Light symptoms group) and MS (Mild symptoms group) and for all 5 sessions; Error bars represent the between-subject standard error 


\section{Simulator sickness in older drivers}

\section{Braking at intersections}

Time to reach the intersection

The mean time to reach the intersection was significantly longer for the MS than the LS group, regardless of the session (Figure 2a, left panel). On average, the participants in the MS group started braking 2 seconds earlier than the participants in the LS group. The ANOVA yielded a main effect of Group $(F(1,14)=5.6, p=0.01)$. The main effect of Session and the interaction of Group by Session were not significant $(p>0.05)$.

The MS group also exhibited larger variability than the LS group during the pre-test whereas no significant difference was observed between groups during the post-test (Figure 2a, right panel). This is supported by a significant interaction of GroupxSession: $F(1,14)=1.4, p<0.05)$. Neither the main effects of Group or Session showed a significant difference $(p>0.05)$.

\section{Time of deceleration}

Participants in the MS group had longer time of deceleration than those from the LS group for both the pre-test and the post-test (Figure $2 b$, left panel). Note that the time of deceleration decreased between pre-test and post-test for both the MS and the LS groups. These observations are supported by main effects of Group: $F(1,14)=1.4$, $p<0.05$ and Session: $F(1,14)=6.3, p<0.05$. The interaction of GroupxSession did not reach significant level $(p>0.05)$.

The MS group also showed greater time of deceleration variability than the LS group during the pre-test. (Figure $1 \mathrm{~b}$, right panel). This difference vanished during the post-test. The ANOVA showed a significant interaction of GroupxSession: $F(1,14)=5.0, p<0.05)$. The main effects of Group and Session were not significant $(\mathrm{p}>0.05)$.

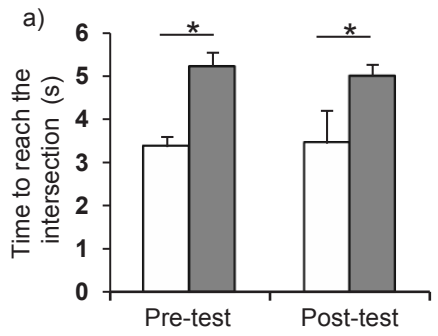

b)
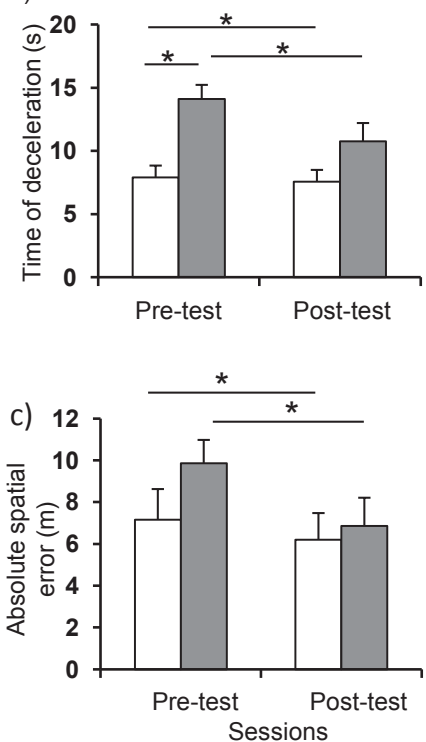
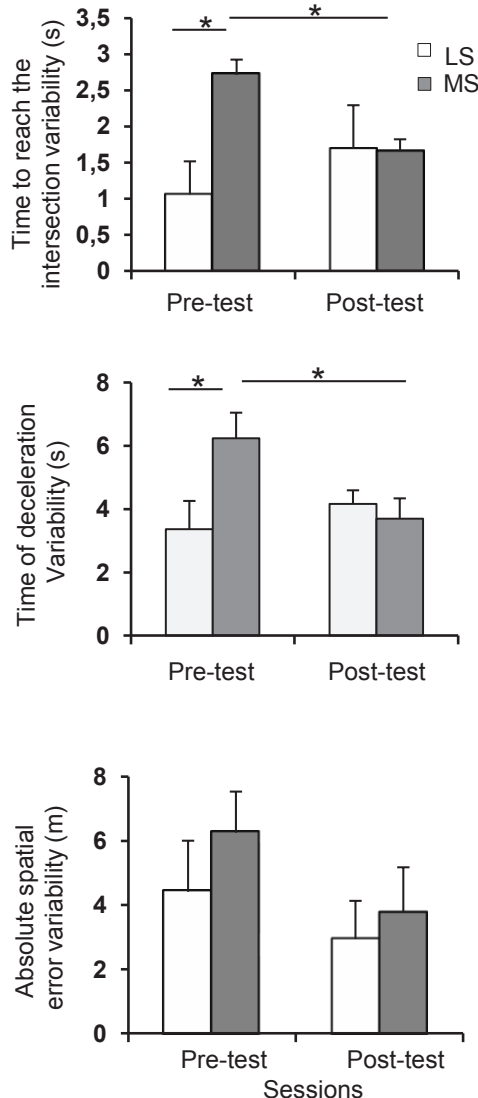

Figure 2. Means (right column) and variability (left column) for (a) time to reach the intersection (upper line), (b) time of deceleration (middle line), and (c) absolute spatial error for both groups and for both pre-test and post-test sessions (lower line); Error bars represent the between-subject standard error; LS=Light symptoms group; $M S=$ Mild symptoms group

\section{Spatial error}

A significant reduction of the absolute spatial error between the pre-test $(8.5 \mathrm{~m})$ and the post-test $(6.5 \mathrm{~m})$ was noted (Figure 2c). This is supported by a significant main effect of Session: $\mathrm{F}(1,14)=6.9$, $\mathrm{p}=0.02$. Greater errors were observed at the pretest for the MS group, but this effect did not reach a significance level as both the main effect of Group and the interaction were not significant $(p>0.05)$. The ANOVA for the variability on the spatial error showed no significant effect ( $p>0.05$ for all effects).

Number of peaks in the jerk-time curves

When approaching an intersection, a smoother decrease of the speed implies a smaller number of peaks in the jerk-time curves. To quantify the smoothness of the braking dynamics, the mean number of peaks in the jerk-time curves and the variability in the number of peaks were analyzed (Figure 3). 


\section{Simulator sickness in older drivers}
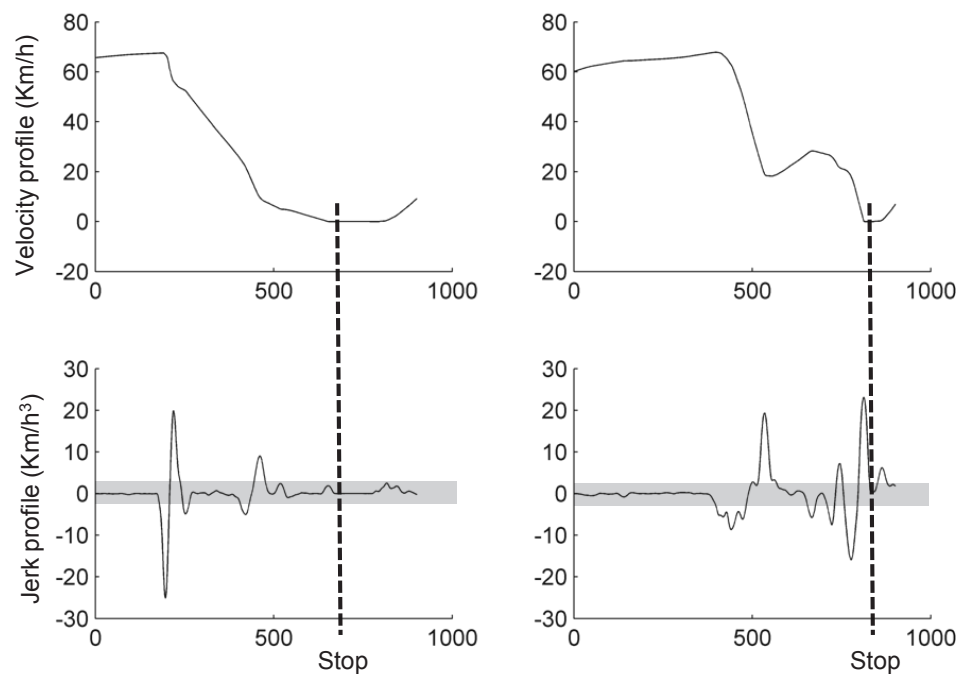

$p<0.05$. The main effect of Group was not significant ( $>>0.05)$.

Gas and brake during deceleration

Repeated and alternated activation of the gas and brake pedals increases the irregularities in the deceleration profile. To evaluate if the number of gas and brake pedals activation differed between the MS and the LS group during the pre-test, a rank test was performed on the total number of activations of the gas and brake pedals. A Mann-Whitney test indicated that the me-

Figure 3. (a) Velocity profile as a function of time, and (b) its corresponding jerk time profile; The left column presents a smooth approach to the intersection whereas the right column depicts a jerkier approach to the intersection; Shadow represents the $\pm 3 \mathrm{~km} / \mathrm{h} 3$ criteria used to calculate the number of peaks in the jerk time profile; Five peaks can be observed for the individual from the LS group and 10 peaks are observed for the individual from the MS group

dian value for the MS group (5.3) was greater than that for the LS group (4.0) and the two groups differed significantly (Mann-Withney $U=56$, $\mathrm{p}<0.05)$. A Wilcoxon $\mathrm{W}$ test

Participants in the MS group showed a greater number of peaks than the LS group both for the pre-test and the post-test sessions (Figure 4a). The ANOVA yielded a main effect of Group: $F(1,14)=6.0, p<0.05)$. The main effect of Session and the interaction of GroupxSession were not significant, $p>0.05$.

As to the variability in the number of peaks, the MS group showed a greater variability at the pretest than at the post-test whereas the LS group showed a similar variability for both sessions (Figure $4 b$ ). The ANOVA showed a significant main effect of Session: $F(1,14)=5.4, p<0.05$, as well as an interaction of GroubxSession:F(1.14)=9.3. for paired sample showed the MS group did not reduce the total number of pedal activations through repeated exposure (pre-test vs. post-test; $\mathrm{W}(8)=(19), \mathrm{p}>0.05$, one-tailed test).

\section{Discussion}

\section{Differences between MS and LS}

In the present paper we evaluated whether older participants reporting more pronounced simulator sickness symptoms exhibited differences in their postural stability and braking responses than participants with light or no symptoms. Previously, Stoffregen et al. ${ }^{20}$ have demonstrated that postural instability, measured as head and torso displacements when standing, preceded

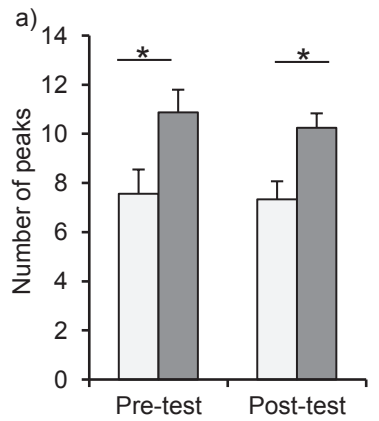

Sessions

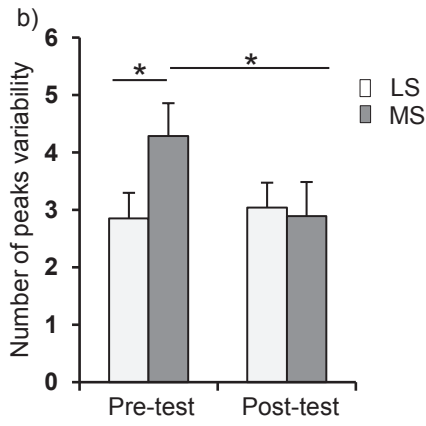

Sessions

Figure 4. (a) Means number of peaks in the jerk-time curves, and (b) variability in the number of peaks in the jerk-time curves for both groups, LS (Light symptoms group) and MS (Mild symptoms group), and for both pre-test and post-test sessions; Error bars represent the between-subject standard error the appearance of motion sickness symptoms and increased with prolonged exposure to nauseogenic stimuli ${ }^{35}$. In the present paper, compared with the LS group, mean head range of motion and variability of the participants who expressed mild simulator symptoms showed only a weak trend toward increased instability. Large between-subject variability explained the lack of significance between groups and sessions. It is noteworthy that torso movements were restrained (if not eliminated) by the back support of the car seat, possibly enhancing head postural stability while 


\section{Simulator sickness in older drivers}

driving. As well, the driving conditions were programmed to attenuate as much as possible nauseogenic conditions (for instance, by avoiding sharp curves and hilly terrain). Furthermore, it is likely that the nauseogenic situations were self-generated by the drivers rather than resulting from the driving condition per se (for instance, through repeated activation/deactivation of the gas and brake pedals when braking at intersections). On the contrary, in Stoffregen et al. task, participants were either standing or sitting on a stool without back support and the visual stimuli aimed at increasing the nauseogenic conditions. This may explain the lack of difference in head movements between the two groups in the present study. We also ran an additional analysis (not presented in the Results section) to examine if the head movements for the entire simulation would show greater displacements for the MS group. This analysis also yielded an absence of difference between the MS and the LS group. Perhaps, asking participants to get out of the simulator and stand on a force platform at regular intervals during the simulation could allow highlighting postural instability for individuals reporting greater sickness symptoms. This would certainly deserve some thoughts if such a procedure could permit to identify individuals susceptible to report symptoms as the duration of the exposure to the simulation increases. At this point, however, our analyses of head displacements do not support the suggestion that individuals expressing greater symptoms also exhibit greater postural instability (as measured from head displacements).

Significant differences between groups emerged for the braking responses. We observed that during the pre-test, participants within the MS group started braking earlier, had longer time of deceleration and were more variable than the LS group. Because the time to reach the intersection is a measure of temporal prediction based on the visual information available, this suggests that participants who felt more pronounced symptoms had more difficulties to process the visual information presented during the simulation to estimate the time to reach the stop line. It has been proposed that one factor influencing temporal estimation in visually simulated environments is the perception of distance ${ }^{36,37}$. Poorer accuracy at the stop line was noted for the MS group, but the difference with the LS group did not reach significance. The greater number of adjustments observed during the braking responses from the MS group (i.e., more peaks in the jerk-time profile and more activation of the gas and brake pedals during the deceleration) also suggests difficulties in estimating the distance and the time of arrival at the stop line.
Recent motor control models propose that visual information can be used continuously to amend the arm trajectory with minimal jerk and time cost. Based on prediction mechanisms, the adjustments are smoothly imbedded in the trajectory allowing overcoming the neural delay in processing visual information. However, when predictions are less accurate, the close-loop control must be relied on and is weighted strongly ${ }^{38}$. Inherent to the close-loop control, is that the feedback from the response (i.e., visual flow from the braking in the current study) is processed following long sensory delay. When the desired state is not reach (e.g., proper deceleration rate needed to stop precisely at the intersection), a novel response is implemented. Any additional adjustment to reach a desire state requires additional pedal activations and increases the jerk. This suggest the MS group might have had more difficulties making accurate predictions, suggesting that participants in the MS group relied more heavily on close-loop control than participants in the LS group. The participants in the MS group also exhibited more variability in estimating the time to reach the intersection - interpreted as poor predictive efficiency - which also supports this interpretation. In accordance, larger incidence of simulator sickness found in older participants might be related with decline in motor prediction ability that is observed with aging ${ }^{39}$.

\section{Adaptation processes}

Through repeated exposure to the simulator ( 5 sessions), the VAS and the SSQ total score decreased gradually from pre-test to post-test for the MS group, but remained slightly higher than scores observed for the LS group for the posttest. The decrease in VAS and SSQ scores can be interpreted as evidence of an adaptation (i.e., alleviating the simulator sickness symptoms). Unexpectedly, however, we found little support suggesting this adaptation was associated to any change in postural stability and braking smoothness. For the four variables analyzed (time to reach the intersection, time of deceleration, spatial error and number of peaks in the jerk-time curves and number of gas and brake pedals activation), the MS group did not show larger improvement than the LS group (none of the interactions of GroupxSession were significant). Strikingly, however, a larger decrease in variability was observed for the MS group for 3 out of 4 variables (time to reach the intersection, time of deceleration, and number of peaks in the jerktime curves). Because the variability was calculated on five intersections, the larger variability observed for the MS group might result from outlier data. However, none of the participants showed outlier data (that is, a value above 1.2 standard deviation from the mean), regardless of 


\section{Simulator sickness in older drivers}

the variable. Rather, it seems that the larger variability for the MS group originated from participants producing more variable responses across intersections. In the same vein, reduction of variability with exposure likely resulted from participants producing more consistent responses on the different variables.

If we assume that a sensory conflict is at the basis of the simulator sickness observed when driving in a fixed-based simulator, adaptation to simulator sickness might not exclusively involve the acquisition of new braking responses or new driving behaviors. When the central nervous system faces conflicting sensory information, sensory adaptation sometimes consists of selectively suppressing reafference (i.e., sensory feedback) ${ }^{40-42}$. For instance, the deactivation of the parieto-insular vestibular cortex (i.e., cortical area responsible for vestibular perception) was observed in presence of visuo-vestibular conflict (i.e., circular vection ${ }^{41}$ ). Conversely, a significant deactivation of the visual cortex was found during vestibular stimulation ${ }^{43}$. Known as the reciprocal inhibitory visual-vestibular interaction, this mechanism might protect visual perception of self-motion from potential vestibular mismatches caused by a simulated environment. As well, this mechanism could allow the perception of self-motion to shift from one sensory modality to the other. Although visual information is not perfectly suited for deceleration control, inhibitory visual-vestibular interaction might permit to weight more importantly the visual information when driving in a simulator and, thus, alleviating the mismatch between expected and actual sensory information. This transitory state might allow attenuating the symptoms.

\section{Prevention of simulator sickness}

In order to reduce simulator sickness symptoms, the simulation sessions were design according to previous recommendations ${ }^{44}$, such as, the simulator room was maintained at a relatively cold temperature $\left(19.5^{\circ} \mathrm{C}\right)$ and the presentation of the visual information was refreshed at a rate between 25 to $30 \mathrm{~Hz}$. In addition, before each session, participants were asked if they were in their usual state of fitness (that is, not suffering from a cold or flu, hangover, etc.). Although several strategies were used to reduced simulator sickness incidence, the long exposure to simulated environment used to evaluate multiple driving behaviors increases the chance of severe discomfort ${ }^{45}$. Therefore, new protocols should be developed to reduce simulator sickness symptoms and to identify participants who will be more prone to be simulator sick. In the present study, we were not able to discriminate between the LS and MS group by looking at the head movement charac- teristics when subjects were seated and driving in the simulator but observed that participants who felt more severe symptom exhibited a different pattern of deceleration. Future protocols should implement a short familiarization session that includes braking behavior, in order to identify participants who are more prone to feel simulator sickness symptoms when experiencing longer exposure. Because our results clearly show that repeated exposures reduce simulator sickness symptoms, as has been reported earlier ${ }^{45}$. A stepby-step training should be implemented for those participants who are more susceptible to feel pronounced discomfort to longer experimental runs. This will allow some adaptation and a decrease of the symptoms before longer experimental runs are presented. Such procedures, although they require several visits from the participants, might offer a new opportunity to prevent/reduce withdrawal of participants in driving simulator experiments, which is known as one of the major factors limiting the widespread use of simulator. To some extent, this adaptation process could be to driving simulator what is known as getting one's sea legs when boating ${ }^{46,47}$.

A popular view that is generally accepted is that the virtual environment should coincide with the reality to reduce motion, simulator or cyber sickness ${ }^{44}$. Recently, however, it has been proposed that inducing incongruence between the virtual and the real world environment yield less severe visually induced sickness symptoms ${ }^{48}$. For instance, sickness symptoms were less pronounced when the external field of view (visual angle between the observer and the screen) did not coincide with the internal field of view (graphic field of view of the real scene) ${ }^{48,49}$. Furthermore, in Bos et al. experiments ${ }^{48,49}$, a more natural perception of walking speed of $5 \mathrm{~km} / \mathrm{h}$ was reach when the simulated walking speed was set at $13.6 \mathrm{~km} / \mathrm{h}$. This suggests that introducing a gain between the speed at which the visual information is presented in the simulation and the reality might contribute to reduce sickness symptoms when driving in a simulator. For instance, when pressing the brake pedal, the visual deceleration could occur faster than in the real world. Howev$\mathrm{er}$, it is not known whether inducing a difference between the virtual environment and the reality during practice in a simulator would impairs the transfer of driving behavior to on-road driving. Obviously, more research is needed to explore different strategies to reduce simulator sickness and their impact on the on-road driving transfer.

\section{Conclusion}

In the present paper we found evidences that participants who were more sensitive to simulator sickness showed distinct behaviors while 


\section{Simulator sickness in older drivers}

braking at intersections. Through exposures, symptoms associated with simulator sickness were reduced and this was accompanied with a reduction of variability in braking related variables. However, we found limited evidence of

\section{Acknowledgement}

Special thanks to all drivers who participated in the project. This project (COBVIS-D) received support from AUTO21 and the Société de l'Assurance Automobile du Québec (SAAQ). ML was supported by a graduate fellowship from the Fonds de recherche du Québec - Santé (FRQS).

\section{References}

1. Fisher DL, Rizzo M, Caird JK, Lee JD. Handbook of Driving Simulation for Engineering, Medicine, and Psychology. 1st editon. Boca Raton: CRC Press; 2011; 751 pp

2. Anibas J. A study of virtual simulation in a truck driver training program. Menomonie: University of Wisconsin-Stout; 2008

3. Zomeren $\mathrm{AH}$ van, Brouwer $\mathrm{WH}$, Rothengatter JA, Snoek JW. Fitness to drive a car after recovery from severe head injury. Archives of Physical Medicine and Rehabilitation 1988;69(2):90-96

4. Logsdon RG, Teri L, Larson EB. Driving and Alzheimer's disease. Journal of General Internal Medicine 1992;7(6):583-588; doi:10.1007\%2FBF02599195

5. Rizzo A, Jounghyun Kim G. A SWOT analysis of the field of virtual reality rehabilitation and therapy. Presence 2005;14(2):119-146; doi:10.1162\%2F1054746053967094

6. Rizzo M, McGehee DV, Dawson JD, Anderson $\mathrm{SN}$. Simulated car crashes at intersections in drivers with Alzheimer disease. Alzheimer Disease and Associated Disorders 2001;15(1):10-20; doi:10. 1097\%2F00002093-200101000-00002

7. Schultheis MT, Rebimbas J, Mourant R, Millis SR. Examining the usability of a virtual reality driving simulator. Assistive Technology 2007;19(1):1-8; quiz 9-10; doi:10.1080\% 2F10400435.2007.10131860

8. Singh H, Barbour BM, Cox DJ. Driving rehabilitation as delivered by driving simulation. Chapter 32 in: Fisher DL, Rizzo M, Caird JK, Lee JD, editors. Handbook of Driving Simulation for Engineering, Medicine, and Psychology. Boca Raton: CRC Press; 2011; pp 1-12

9. Johnson DM. Introduction to and review of simulator sickness research. U.S. Army Research Institute for the Behavioral and Social Sciences Report No.: 1832; 2005 (April)

10. Cassavaugh ND, Domeyer JE, Bakcs RW. Lessons learned regarding simulator sickness in older adult drivers. LNCS 2011;6767:263-269; doi:10.1007\% 2F978-3-642-21666-4_29

11. Caird JK, Edwards CJ, Creaser JI, Horrey WJ. Older driver failures of attention at intersections: using change blindness methods to assess turn decision accuracy. Human Factors 2005;47(2):235-249; doi:10.1518\%2F0018720054679542 braking response adaptation and changes in postural stability. More gradual exposure to simulated environment might be required before evaluating the driving performance of individuals prone to simulator sickness.

12. Kennedy RS, Fowlkes JE. Simulator sickness is polygenic and polysymptomatic: implications for research. International Journal of Aviation Psychology 1992;2(1):23-28; doi:10.1207\%2Fs15327108ij ap0201_2

13. Stoner $\mathrm{H}$ A, Fisher DL, Mollenhauer MJ. Simulator and scenario factors influencing simulator sickness. Chapter 14 in: Fisher DL, Rizzo M, Caird JK, Lee JD, editors. Handbook of Driving Simulation for Engineering, Medicine, and Psychology. Boca Raton: CRC Press; 2011; pp 1-24

14. Lackner JR, Graybiel A. Elicitation of motion sickness by head movements in the microgravity phase of parabolic flight maneuvers. Aviation, Space, and Environmental Medicine 1984;55(6):513-520

15. Reason JT, Brand JJ. Motion sickness. London; Academic Press; 1975; 310 pp

16. Kennedy RS, Graybiel A, McDonough RC, Beckwith FD. Symptomatology under storm conditions in the North Atlantic in control subjects and in persons with bilateral labyrinthine defects. NSAM-928. Research Report, United States Naval School of Aviation Medicine. 1965 (May) pp 1-10; doi:10.3109\%2F00016486809126317

17. Cheung BS, Howard IP, Money KE. Visuallyinduced sickness in normal and bilaterally labyrinthine-defective subjects. Aviation Space and Environmental Medicine 1991;62(6):527-531

18. Riccio GE, Stoffregen TA. Gravitoinertial force versus the direction of balance in the perception and control of orientation. Psychological Review 1990;97(1):135-137; doi:10.1037\%2F\%2F0033295X.97.1.135

19. Smart LJ, Stoffregen TA, Bardy BG. Visually induced motion sickness predicted by postural instability. Human Factors 2002;44(3):451-465; doi:10.1518\%2F0018720024497745

20. Stoffregen TA, Smart LJ. Postural instability precedes motion sickness. Brain Research Bulletin 1998;47(5):437-448

21. Blaauw GJ. Driving experience and task demands in simulator and instrumented car: a validation study. Soesterberg: Institute for Perception TNO; 1980

22. Kaptein NA, Theeuwes J, Horst R van der. Driving simulator validity: some considerations. Transportation Research Record 1996;1550:30-36; doi:10.3141\%2F1550-05

23. Reed MP, Green PA. Comparison of driving performance on-road and in a low-cost simulator using a concurrent telephone dialling task. Ergonomics 1999;42(8):1015-1037; doi:10.1080\%2F001401399185117

24. Boer ER, Kuge N, Yamamura T, editors. Affording realistic stopping behavior: A cardinal challenge for driving simulators. 1st Human-Centered Trans- 


\section{Simulator sickness in older drivers}

portation Simulation Conference; lowa City, IA; 2001

25. Boer ER, Yamamura T, Kuge N, Girshick A, editors. Experiencing the same road twice: A driver centered comparison between simulation and reality. Driving Simulation Conference; Paris, 2000

26. Malaterre G, Frechaux J. Simulation de la conduite automobile : méthodologie d'évaluation des performances et de la validité d'un simulateur. INRETS 1999

27. Pinto M, Cavallo V, Ohlmann T, Espié S, Rogé J, editors. The perception of longitudinal accelerations: What factors influence braking manoeuvers in driving simulators. Driving Simulation Conference; Paris, 2004

28. Siegler I, Reymond G, Kemeny A, Berthoz A, editors. Sensorimotor integration in a driving simulator: contributions of motion cueing in elementary driving tasks. Driving Simulation Conference; Sophia Antipolis; 2001

29. Lavallière $M$, Simoneau M, Laurendeau D, Teasdale N. Active training and driving-specific feedback improve older drivers' visual search prior to lane changes. BMC Geriatrics 2012;12(5); doi:10.1186/1471-2318-12-5

30. Kennedy RS, Lane NE, Berbaum KS, Lilienthal MG. Simulator Sickness Questionnaire: an enhanced method for quantifying simulator sickness. The International Journal of Aviation Psychology 1993;3(3):203-220; doi:10.1207\%2Fs15327108ij ap0303_3

31. Folstein MF, Folstein SE, McHugh PR. Mini-mental state. A practical method for grading the cognitive state of patients for the clinician. Journal of Psychiatric Research 1975;12(3):189-198

32. Goble DJ. Proprioceptive acuity assessment via joint position matching: from basic science to general practice. Physical Therapy 2010;90(8):11761184; doi:10.2522\%2Fptj.20090399

33. Dellon ES, Mourey R, Dellon AL. Human pressure perception values for constant and moving one- and two-point discrimination. Plastic and Reconstructive Surgery 1992;90(1):112-127; doi:10. 1097\%2F00006534-199207000-00017

34. Kennedy RS, Fowlkes JE, Berbaum KS, Lilienthal MG. Use of a motion sickness history questionnaire for prediction of simulator sickness. Aviation Space and Environmental Medicine 1992;63(7):588-593

35. Bonnet CT, Faugloire E, Riley MA, Bardy BG, Stoffregen TA. Motion sickness preceded by unstable displacements of the center of pressure. Human Movement Science 2006;25(6):800-820; doi:10.1016\%2Fj.humov.2006.03.001

36. Kemeny A, Panerai F. Evaluating perception in driving simulation experiments. Trends in Cognitive Sciences 2003;7(1):31-37; doi:10.1016\%2 FS1364-6613\%2802\%2900011-6

37. Panerai F, Droulez J, Kelada J-M, Kemeny A, Balligand E, Favre B, editors. Speed and safety distance control in truck driving: comparison of simulation and real-world environment. Driving Simulation Conference; Sophia Antipolis; 2001

38. Wolpert DM, Ghahramani Z, Jordan MI. An internal model for sensorimotor integration. Science 1995;269(5232):1880-1882; doi:10.1126\%2Fscience.7569931

39. Skoura X, Personnier P, Vinter A, Pozzo T, Papaxanthis C. Decline in motor prediction in elderly subjects: right versus left arm differences in mentally simulated motor actions. Cortex 2008;44(9):1271-1278; doi:10.1016\%2Fj.cortex.2007.07.008

40. Bernier PM, Burle B, Vidal F, Hasbroucq T, Blouin J. Direct evidence for cortical suppression of somatosensory afferents during visuomotor adaptation. Cerebral Cortex 2009;;19(9):2106-2113; doi:10.1093\%2Fcercor\%2Fbhn233

41. Brandt T, Bartenstein P, Janek A, Dieterich M. Reciprocal inhibitory visual-vestibular interaction. Visual motion stimulation deactivates the parietoinsular vestibular cortex. Brain 1998;121(9):17491758; doi:10.1093\%2Fbrain\%2F121.9.1749

42. Probst T, Straube A, Bles W. Differential effects of ambivalent visual-vestibular-somatosensory stimulation on the perception of self-motion. Behavioural Brain Research 1985;16(1):71-79; doi:10.1016/0166-4328(85)90083-X

43. Wenzel R, Bartenstein P, Dieterich M, Danek A, Weindl A, Minoshima S, Ziegler S, Schwaiger $M$, Brandt T. Deactivation of human visual cortex during involuntary ocular oscillations: A PET activation study. Brain 1996;119(1):101-110; doi:10.1093\%2Fbrain\%2F119.1.101

44. Pinto M, Cavallo V, Ohlmann T. The development of driving simulators: Toward a multisensory solution. Le Travail Humain 2008;71(1):62-95; doi:10.3917\%2Fth.711.0062

45. Kennedy RS, Stanney KM, Dunlap WP. Duration and exposure to virtual environments: sickness curves during and across sessions. Presence 2000;9(5):463-472; doi:10.1162\%2F105474600566952

46. Stoffregen TA, Chen FC, Varlet M, Alcantara C, Bardy BG. Getting your sea legs. PLoS One 2013;8(6):e66949; doi:10.1371\%2Fjournal. pone.0066949

47. Turner M, Griffin MJ. Motion sickness incidence during a round-the-world yacht race. Aviation Space and Environmental Medicine 1995;66(9):849-856

48. Emmerik ML van, Vries SC de, Bos JE. Internal and external fields of view affect cybersickness. Displays 2011;32(4):169-174; doi:10.1016\%2Fj. displa.2010.11.003

49. Bos JE, Vries SC de, Emmerik ML van, Groen EL. The effect of internal and external fields of view on visually induced motion sickness. Applied Ergonomics 2010;41(4):516-521; doi:10.1016\%2Fj. apergo.2009.11.007 